\title{
CONFORMAL REPRESENTATIONS OF LEIBNIZ ALGEBRAS
}

\author{
PAVEL KOLESNIKOV
}

\begin{abstract}
In this note we present a more detailed and explicit exposition of the definition of a conformal representation of a Leibniz algebra. Recall (arXiv:math/0611501 v3) that Leibniz algebras are exactly Lie dialgebras. The idea is based on the general fact that every dialgebra that belongs to a variety Var can be embedded into a conformal algebra of the same variety. In particular, we prove that an arbitrary (finite dimensional) Leibniz algebra has a (finite) faithful conformal representation. As a corollary, we deduce the analogue of the PBW-theorem for Leibniz algebras.
\end{abstract}

Introduction. In [1] we studied the relation between conformal algebras [2] (these are algebraic systems with a countable number of multiplications coming from mathematical physics [3]) and dialgebras $[4,5]$ (these are systems with two operations of multiplication that were introduced in relation with Leibniz algebras). We have proposed a general notion of what is a variety of dialgebras generalizing the classes of associative [4] and alternative [6] dialgebras. In particular, Lie dialgebras are exactly Leibniz algebras. The main result of [1] states that an arbitrary dialgebra of a given variety can be embedded (in a canonical way) into a conformal algebra of the same variety.

In this paper, we focus on the embedding of Lie dialgebras (Leibniz algebras) into conformal algebras. For this variety, the construction of a conformal envelope from [1] can be simplified by using the notion of a conformal representation. We will show that every (finite dimensional) Leibniz algebra can be embedded into current conformal algebra over the algebra of linear transformations of a (finite dimensional) linear space. As a corollary, we obtain a new proof of the theorem on injective embedding of a Leibniz algebra into an associative dialgebra and, more explicitly, prove an analogue of the Poincare - Birkhoff-Witt (PBW) theorem for Leibniz algebras [5].

1. Varieties of dialgebras. A dialgebra is a linear space $A$ endowed with two bilinear operations $\dashv, \vdash$. In [4] and [6], the notions of associative and alternative dialgebras were introduced. These definitions were motivated by the relations between dialgebras and Leibniz algebras. In particular, the associativity identities for dialgebras are chosen in such a way that the new operation

$$
[a b]=a \vdash b-b \dashv a
$$

makes a dialgebra $A$ to be a left Leibniz algebra, i.e., the identity

$$
\left[x_{1}\left[x_{2} x_{3}\right]\right]=\left[\left[x_{1} x_{2}\right] x_{3}\right]+\left[x_{2}\left[x_{1} x_{3}\right]\right]
$$

holds. The Leibniz algebra $A^{(-)}$obtained is an analogue of the adjoint Lie algebra of an associative algebra.

Partially supported by RFBR. The author gratefully acknowledges the support from Deligne 2004 Balzan prize in mathematics. 
In [1], we developed a general approach to the definition of what is a variety of dialgebras via the notion of an operad. In terms of identities, this definition can be stated as follows.

DEFINITION 1. Let Var be a variety of algebras defined by a family $\Sigma$ of polylinear identities. A dialgebra $A$ is said to be a Var-dialgebra, if it satisfies the identities

$$
\begin{gathered}
\left(x_{1} \dashv x_{2}\right) \vdash x_{3}-\left(x_{1} \vdash x_{2}\right) \vdash x_{3}, \quad x_{1} \dashv\left(x_{2} \vdash x_{3}\right)-x_{1} \dashv\left(x_{2} \dashv x_{3}\right), \\
t_{i}\left(x_{1}, \ldots, x_{n}\right), \quad t \in \Sigma, \operatorname{deg} t=n, i=1, \ldots, n,
\end{gathered}
$$

where $t_{i}$ is obtained from $t$ by replacing of each monomial $\left(x_{j_{1}} \ldots x_{j_{n}}\right)$ with $\left(x_{j_{1}} \vdash\right.$ $\left.\cdots \vdash x_{i} \dashv \ldots \dashv x_{j_{n}}\right)$ preserving the bracketing.

If $\Sigma=\left\{\left(x_{1} x_{2}\right) x_{3}-x_{1}\left(x_{2} x_{3}\right)\right\}$ (associativity) then the system of identities (2) coincides with the one introduced in [4]:

$$
\begin{gathered}
t_{1}=\left(x_{1} \dashv x_{2}\right) \dashv x_{3}-x_{1} \dashv\left(x_{2} \dashv x_{3}\right), \quad t_{2}=\left(x_{1} \vdash x_{2}\right) \dashv x_{3}-x_{1} \vdash\left(x_{2} \dashv x_{3}\right), \\
t_{3}=\left(x_{1} \vdash x_{2}\right) \vdash x_{3}-x_{1} \vdash\left(x_{2} \vdash x_{3}\right) .
\end{gathered}
$$

If $\Sigma=\left\{x_{1} x_{2}+x_{2} x_{1}, x_{1}\left(x_{2} x_{3}\right)-x_{2}\left(x_{1} x_{3}\right)-\left(x_{1} x_{2}\right) x_{3}\right\}$ (Lie algebra identities) then, with respect to the operation $[x y]=x \vdash y$, the identities (2) are equivalent to the left Leibniz identity (1). Conversely, an arbitrary (left) Leibniz algebra with respect to $x \vdash y=[x y], x \dashv y=-[y x]$ is a Lie dialgebra in the sense by Definition 1 .

2. Conformal algebras. Let $H$ be a cocommutative Hopf algebra with coproduct $\Delta$, counit $\varepsilon$ and antipode $S$. We will use the Sweedler notation [7] omitting the summation symbols, e.g.,

$$
\begin{gathered}
\Delta(h)=h_{(1)} \otimes h_{(2)}, \quad(\Delta \otimes \mathrm{id}) \Delta(h)=h_{(1)} \otimes h_{(2)} \otimes h_{(3)}, \\
(S \otimes \mathrm{id}) \Delta(h)=h_{(-1)} \otimes h_{(2)}, \quad(\mathrm{id} \otimes S) \Delta(h)=h_{(1)} \otimes h_{(-2)} .
\end{gathered}
$$

Definition $2[2,8,9]$. Let $M$ and $N$ be two left $H$-modules. A conformal linear map from $M$ to $N$ is a linear map $a$ from $M$ to $H^{\otimes 2} \otimes_{H} N$ such that $a(h u)=$ $\left(1 \otimes h \otimes_{H} \mathrm{id}\right) a(u), h \in H, u \in M$. Hereinafter, $H^{\otimes 2}=H \otimes H$ is considered as the outer product of regular right $H$-modules.

Note that $H \otimes H$ is a free right $H$-module with a basis $\left\{h_{i} \otimes 1\right\}$, where $\left\{h_{i}\right\}$ is a linear basis of $H$ over $\mathbb{k}$. Indeed, $f \otimes g=\left(f g_{(-1)} \otimes 1\right) g_{(2)}=\sum_{i}\left(h_{i} \otimes 1\right) g_{i}$, and such a presentation is unique.

The space of all conformal linear maps from $M$ to $N$ is denoted by $\operatorname{Chom}(M, N)$. If $N=M$ then we use Cend $M$ for $\operatorname{Chom}(M, M)$.

Let us define the structure of a left $H$-module on $\operatorname{Chom}(M, N)$ as follows:

$$
(h a)(u)=\left(h \otimes 1 \otimes_{H} \mathrm{id}\right) a(u), \quad h \in H, a \in \operatorname{Chom}(M, N), u \in M .
$$

Definition $3[9,10]$. A left $H$-module $C$ endowed with an $H$-linear map $\mu: C \rightarrow$ Cend $C$ is called an $H$-conformal algebra (or pseudo-algebra over $H$ ). The operation $(a * b)=\mu(a) b \in H^{\otimes 2} \otimes_{H} C, a, b \in C$, is called pseudo-product.

If $H=\mathbb{k}$ then the notion of an $H$-conformal algebra coincides with the notion of an ordinary algebra over a field. If $H=\mathbb{k}[T]$ (with respect to the canonical Hopf algebra structure) and char $\mathbb{k}=0$ then an $H$-conformal algebra is exactly what is called by conformal algebra in [2]. In this paper, we will preferably consider $H$-conformal algebras over $H=\mathbb{k}[T]$ without a restriction on the characteristic of $\mathbb{k}$. 
The simplest example of an $H$-conformal algebra is provided by the construction of the current algebra Cur $A$ over an ordinary algebra $A$. Given an algebra $A$, one may construct Cur $A$ as the free $H$-module $H \otimes A$ endowed with the $H$-linear map

$$
\mu(f \otimes a): h \otimes b \mapsto f \otimes h \otimes_{H}(1 \otimes a b), \quad a, b \in A, f, h \in H .
$$

The pseudo-product $*: C \otimes C \rightarrow(H \otimes H) \otimes_{H} C$ on an $H$-conformal algebra $C$ is an $H^{\otimes 2}$-linear map. A categorical interpretation of the notion of an algebra over an operad implies the following generalization (see [10]): the pseudo-product can be considered as a map from $\left(H^{\otimes n} \otimes_{H} C\right) \otimes\left(H^{\otimes m} \otimes_{H} C\right)$ to $H^{\otimes(n+m)} \otimes_{H} C$ given by

$$
\left(F_{1} \otimes_{H} a_{1}\right) *\left(F_{2} \otimes_{H} a_{2}\right)=\left(F_{1} \otimes F_{2} \otimes_{H} 1\right)\left(\Delta^{n-1} \otimes \Delta^{m-1} \otimes_{H} \text { id }\right)\left(a_{1} * a_{2}\right),
$$

where $F_{1} \in H^{\otimes n}, F_{2} \in H^{\otimes m}, a_{1}, a_{2} \in C, \Delta^{0}(h)=h, \Delta^{k}(h)=h_{(1)} \otimes \ldots \otimes h_{(k+1)}$ for $h \in H, k \geq 1$.

Relation (3) allows to interpret an arbitrary polylinear term $t$ (in the signature of an algebra over a field) as a function on $C^{\otimes n}(n=\operatorname{deg} t)$ with values in $H^{\otimes n} \otimes_{H} C$. Definition 4 [10]. Let Var be a variety of algebras defined by a family $\Sigma$ of polylinear identities. An $H$-conformal algebra $C$ over a cocommutative Hopf algebra $H$ belongs to a variety Var (is a Var-pseudo-algebra, for short) if it satisfies the identities

$$
t^{*}\left(x_{1}, \ldots, x_{n}\right), \quad t \in \Sigma,
$$

where $t^{*}$ is obtained from $t$ by replacing each monomial $\left(x_{1 \sigma} \ldots x_{n \sigma}\right), \sigma \in S_{n}$, with $\left(\sigma \otimes_{H}\right.$ id $)\left(x_{1 \sigma} * \cdots * x_{n \sigma}\right)$ preserving the bracketing. Here $\sigma$ acts on $H^{\otimes n}$ by the rule $\left(h_{1} \otimes \cdots \otimes h_{n}\right)^{\sigma}=\left(h_{1 \sigma^{-1}} \otimes \cdots \otimes h_{n \sigma^{-1}}\right)$.

REMARK. The class of Var-pseudo-algebras over a Hopf algebra $H$ is not a variety in the ordinary sense: it is not closed under infinite direct products.

On every $H$-conformal algebra $C$ one may define the operations $\dashv$, $\vdash$ by the following rule: if $a * b=\sum_{i} h_{i} \otimes 1 \otimes_{H} c_{i}, a, b, c_{i} \in C, h_{i} \in H$, then

$$
a \vdash b=\sum_{i} \varepsilon\left(h_{i}\right) c_{i}, \quad a \dashv b=\sum_{i} h_{i} c_{i} .
$$

The dialgebra obtained is denoted by $C^{(0)}$.

The main result of [1] can be stated in the following form.

Theorem 1. If $C$ is a Var-pseudo-algebra then $C^{(0)}$ is a Var-dialgebra. Conversely, an arbitrary Var-dialgebra can be embedded into $C^{(0)}$ for an appropriate Varpseudo-algebra $C$ over $H=\mathbb{k}[T]$.

3. Faithful representations of Leibniz algebras. Let $H$ be the coordinate Hopf algebra of an abelian linear algebraic group $G$. Let us fix a basis $\left\{h_{i}\right\}$ of $H$ over $\mathbb{k}$. Consider three left $H$-modules $M, N$, and $V$ together with an $H^{\otimes 2}$-linear map

$$
*: M \otimes N \rightarrow H^{\otimes 2} \otimes_{H} V .
$$

Define the family of operations $\left(\cdot{ }_{(z)} \cdot\right): M \otimes N \rightarrow V, z \in G$, as follows. If $u * v=\sum_{i} h_{i} \otimes 1 \otimes_{H} w_{i}$ for $u \in M, v \in N$, then

$$
\left(u_{(z)} v\right)=\sum_{i} h_{i}\left(z^{-1}\right) w_{i} \in V
$$


The family of bilinear operations constructed does not depend on the choice of $\left\{h_{i}\right\}$, and it has the following properties:

(C1) for every $u, v$ the map $x \mapsto\left(u_{(x)} v\right)$ is a regular $V$-valued function on $G$;

(C2) $\left(h u_{(z)} v\right)=h\left(z^{-1}\right)\left(u_{(z)} v\right)$;

(C3) $\left(u_{(z)} h v\right)=h_{(1)}(z) h_{(2)}\left(u_{(z)} v\right)$.

The property (C1) means that for every $u \in M, v \in N$ there exists a uniquely defined (if a basis $\left\{h_{i}\right\}$ is fixed) finite set of $w_{i} \in V$ such that $\left(u_{(z)} v\right)=\sum_{i} h_{i}\left(z^{-1}\right) w_{i}$ for all $z \in G$. In this settings, define $\left\{u_{(z)} v\right\}=\sum_{i} h_{i(1)}(z) h_{i(2)} w_{i}$.

The structure of an $H$-conformal algebra on a left $H$-module $C$ is completely defined by the family of operations (4) for $M=N=V=C$. The identities of conformal algebras described in Definition 4 can be expressed in terms of operations $\left(\cdot{ }_{(z)} \cdot\right)$ and $\left\{\cdot{ }_{(z)} \cdot\right\}$. For example, an $H$-conformal algebra $C$ is associative if and only if

$$
a_{(z)}\left(b_{(y)} c\right)=\left(a_{(z)} b\right)_{(y z)} c, \quad a, b, c \in C, z, y \in G .
$$

REMARK. If $G=\mathbb{A}_{1} \simeq(\mathbb{k},+)$, char $\mathbb{k}=0$ then $(\cdot(z) \cdot)$ coincides with what is called $\lambda$-bracket in [8].

If $M$ is a left $H$-module then the action of Cend $M$ on $M$ is also completely defined by the family of $G$-products $\left(\cdot{ }_{(z)} \cdot\right)$ : Cend $M \otimes M \rightarrow M, z \in G$, built by the map $a * u=a(u), a \in$ Cend $M, u \in M$. Define similar operations $(\cdot(z) \cdot)$ and $\{\cdot(z) \cdot\}, z \in G$, on the space Cend $M$ by the following rule: if $a, b \in \operatorname{Cend} M$, $u \in M$,

$$
b(u)=\sum_{i} h_{i} \otimes 1 \otimes_{H} v_{i}, \quad a\left(v_{i}\right)=\sum_{j} h_{j} \otimes 1 \otimes_{H} w_{i j},
$$

then

$$
\left(a_{(z)} b\right)(u)=\sum_{i, j} h_{j}\left(z^{-1}\right) h_{i(1)}(z) h_{i(2)} \otimes 1 \otimes_{H} w_{i j}
$$

and

$$
\left\{a_{(z)} b\right\}(u)=\sum_{i, j} h_{i}(z) h_{j(1)}\left(z^{-1}\right) h_{j(2)} \otimes 1 \otimes_{H} w_{i j} .
$$

(It is easy to check that $\left(a_{(z)} b\right)$ and $\left\{a_{(z)} b\right\}$ belongs to Cend $M$ for all $z \in G$.) The operations (6) on Cend $M$ satisfy the relations (C2), (C3), and (5), but (C1) fails in general.

Proposition 2. The linear space Cend $M$ with respect to the operations $a \vdash b=$ $\left(a_{(e)} b\right), a \dashv b=\left\{a_{(e)} b\right\}$, where $e$ is the unit of $G$, is an associative dialgebra denoted by Cend $M^{(0)}$. Proof. As we have already mentioned, the operations (6) on Cend $M$ satisfy (5). Moreover, the following identities hold:

$$
\begin{aligned}
& \left\{\left\{a_{(z)} b\right\}_{(y)} c\right\}=\left\{a_{(y z)}\left\{b_{(y)} c\right\}\right\}=\left\{a_{(y z)}\left(b_{\left(z^{-1}\right)} c\right)\right\}, \\
& \left(\left\{a_{(z)} b\right\}_{(y)} c\right)=\left(\left(a_{(y z)} b\right)_{(y)} c\right)=\left(a_{(y z)}\left(b_{\left(z^{-1}\right)} c\right)\right)
\end{aligned}
$$

for all $y, z \in G$.

Indeed, the definitions (6) and (7) are chosen in such a way that

$$
\left(a_{(z)} b\right)_{(w)} u=a_{(z)}\left(b_{\left(w z^{-1}\right)} u\right), \quad\left\{a_{(z)} b\right\}_{(w)} u=a_{(w z)}\left(b_{\left(z^{-1}\right)} u\right)
$$

for $a, b \in$ Cend $M, u \in M, z, w \in G$. These relations imply (8), (9), and also (5), e.g.,

$$
\left\{\left\{a_{(z)} b\right\}_{(w)} c\right\}_{(y)} u=\left\{a_{(z)} b\right\}_{(w y)}\left(c_{\left(w^{-1}\right)} u\right)=a_{(w y z)}\left(b_{\left(z^{-1}\right)}\left(c_{\left(w^{-1}\right)} u\right)\right)
$$


and

$\left\{a_{(w z)}\left\{b_{(w)} c\right\}\right\}_{(y)} u=a_{(w y z)}\left(\left\{b_{(w)} c\right\}_{\left((w z)^{-1}\right)} u\right)=a_{(w y z)}\left(b_{\left(z^{-1}\right)}\left(c_{\left(w^{-1}\right)} u\right)\right)$.

Other equations in (8), (9) can be deduced analogously.

If $y=z=e$ then (5), (8), (9) turn into the identities of an associative dialgebra.

The adjoint Lie dialgebra (Leibniz algebra) with the operation $[a b]=\left(a_{(e)}\right.$ $b)-\{b(e) a\}, a, b \in$ Cend $M$, is denoted by gc $M^{(0)}$.

Definition 5. A conformal representation of an associative (resp., Lie) dialgebra $A$ on a left $H$-module $M$ is a homomorphism of dialgebras $\rho: A \rightarrow C^{(0)}$, where $C=$ Cend $M$ (resp., gc $M$ ). If $M$ is a finitely generated $H$-module then $\rho$ is said to be a finite representation.

In the case of ordinary algebras for $G=\{e\}$ Definition 5 coincides with the usual definition of what is a representation of an algebra on a linear space. By the classical Poincare - Birkhoff-Witt and Ado-Iwasawa theorems an arbitrary (finite dimensional) Lie algebra has a (finite dimensional) faithful representation. A Leibniz algebra which is not a Lie algebra obviously has no faithful representation for $G=\{e\}$ (as well as for any finite group), but it turns out that a faithful representation exists for affine line $G=\mathbb{A}_{1}$.

Theorem 3. An arbitrary (finite dimensional) Leibniz algebra has a (finite) faithful conformal representation for $G=\mathbb{A}_{1}$.

Proof. Let $L$ be a Leibniz algebra, $L^{\text {alg }}=L / \operatorname{Span}\{[a b]+[b a] \mid a, b \in L\}$ is the ordinary Lie algebra. Denote the image of an element $a \in L$ in $L^{\text {alg }}$ by $\bar{a}$.

Suppose we have fixed a non-trivial $L^{\text {alg }}$-module $V$, and consider the space $M_{0}=$ $V \oplus(L \otimes V)$.

Recall that the coordinate Hopf algebra of $G=\mathbb{A}_{1}$ is the polynomial algebra $H=\mathbb{k}[T]$, where $\Delta(h)=h(T \otimes 1+1 \otimes T), \varepsilon(h)=h(0), S(h)=h(-T)$ for $h \in H$. We will construct a homomorphism $\rho: L \rightarrow \operatorname{gc} M^{(0)}$ for $M=H \otimes M_{0}$. To define a conformal linear map $\rho(a), a \in L$, it is enough to fix the values of $\left(\rho(a)_{(z)} u\right) \in M$, $z \in \mathbb{k}, u \in M_{0}$. Set

$$
\begin{gathered}
\rho(a)_{(z)} v=\bar{a} v+z(a \otimes v), \quad v \in V, \\
\rho(a)_{(z)}(b \otimes v)=b \otimes \bar{a} v+[a b] \otimes v, \quad b \in L, v \in V .
\end{gathered}
$$

Relations (10) define an injective $H$-linear map from $L$ to Cend $M$. Let us show that $\rho$ is a conformal representation of the Leibniz algebra $L$. It is enough to check that

$$
\rho([a b])=\left(\rho(a)_{(0)} \rho(b)\right)-\left\{\rho(b)_{(0)} \rho(a)\right\}
$$

for all $a, b \in L$. Indeed, using (5) and (9) we obtain

$$
\begin{gathered}
\left(\left(\rho(a)_{(0)} \rho(b)\right)-\left\{\rho(b)_{(0)} \rho(a)\right\}\right)_{(z)} v=\rho(a)_{(0)}\left(\rho(b)_{(z)} v\right)_{-\rho(b)_{(z)}\left(\rho(a)_{(0)} v\right)} \\
=\rho(a)_{(0)}(\bar{b} v+z(b \otimes v))-\rho(b)_{(z)}(\bar{a} v) \\
=\bar{a} \bar{b} v+z(b \otimes \bar{a} v)+z[a b] \otimes v-\bar{b} \bar{a} v-z b \otimes \bar{a} v \\
=[\bar{a}, \bar{b}] v+z[a b] \otimes v=\rho([a b])_{(z)} v
\end{gathered}
$$




$$
\begin{aligned}
& \left(\left(\rho(a)_{(0)} \rho(b)\right)-\left\{\rho(b)_{(0)} \rho(a)\right\}\right)_{(z)}(c \otimes v) \\
& =\rho(a)_{(0)}(c \otimes \bar{b} v+[b c] \otimes v)-\rho(b)_{(z)}(c \otimes \bar{a} v+[a c] \otimes v) \\
& \quad=c \otimes[\bar{a}, \bar{b}] v+[[a b] c] \otimes v=\rho([a b])_{(z)}(c \otimes v)
\end{aligned}
$$

for $c \in L, v \in V$.

If $\operatorname{dim} L<\infty$ then $\operatorname{dim} M_{0}$ can be chosen to be finite dimensional, and in this case $\rho$ is a finite representation.

It is clear from (10) that the images of $a \in L$ under $\rho$ belong to Cur End $M_{0} \subset$ Cend $M$. Indeed, $\rho(a)=1 \otimes a_{0}-T \otimes a_{1}$, where

$$
\begin{gathered}
a_{0}(v)=\bar{a} v, \quad a_{0}(b \otimes v)=b \otimes \bar{a} v+[a b] \otimes v, \\
a_{1}(v)=a \otimes v, \quad a_{1}(b \otimes v)=0
\end{gathered}
$$

for all $b \in L, v \in V$.

Corollary 4. Every (finite dimensional) Leibniz algebra can be embedded into current conformal algebra over the ordinary algebra of linear transformations of a (finite dimensional) linear space.

Corollary 5 [5]. For every Leibniz algebra $L$ there exists an associative dialgebra $A$ such that $L \subset A^{(-)}$.

In the last statement, just consider $A=\left(\operatorname{Cur} \text { End } M_{0}\right)^{(0)}$.

Let $L$ be a Leibniz algebra. Denote by $U(L)$ its universal enveloping associative dialgebra built in $[4,5]$. Recall that $U(L)$ is a quotient of the free associative dialgebra $F(L)$ generated by the space $L$ by the ideal generated in $F(L)$ by all elements of the form $a \vdash b-b \dashv a-[a b], a, b \in L$. We will use $\tilde{c}$ to denote the image of $c \in L \subset F(L)$ in $U(L)$.

It follows from the universal property of $U(L)$ that an arbitrary conformal representation $\rho: L \rightarrow \operatorname{gc} M^{(0)}=\left(\text { Cend } M^{(0)}\right)^{(-)}$induces a representation $U(\rho)$ : $U(L) \rightarrow$ Cend $M^{(0)}$. of the associative dialgebra $U(L)$. If $M$ is the module from Theorem 3 built on the $L^{\text {alg }}$-module $V=U\left(L^{\text {alg }}\right)$ then let us denote the corresponding conformal representation $L \rightarrow \operatorname{gc} M^{(0)}$ by $\rho_{U}$.

Theorem 6. The representation $U\left(\rho_{U}\right)$ is faithful.

Proof. We will prove the theorem without a reference to the results of $[4,5]$.

Let us fix a linear basis $B$ of $L$ in the following form: $B=\left\{a_{i} \mid i \in I\right\} \cup\left\{b_{j} \mid\right.$ $j \in J\}$, where $\left\{\bar{a}_{i} \mid i \in I\right\}$ is a basis of $L^{\text {alg }},\left\{y_{j} \mid j \in J\right\}$ is a basis of the kernel of

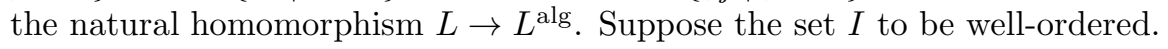

Lemma 7. The elements of the form

$$
\tilde{c} \dashv \tilde{a}_{i_{1}} \dashv \cdots \dashv \tilde{a}_{i_{n}}, \quad c \in B, i_{1} \leq \cdots \leq i_{n}, \quad n \geq 0,
$$

$\operatorname{span} U(L)$.

Proof. Note that the bracketing in (11) is not essential since the operation $\dashv$ is associative.

By making use of the identities of associative dialgebras and the rewriting rule $\tilde{x} \vdash \tilde{y}=\tilde{y} \dashv \tilde{x}+\widetilde{[x y]}, x, y \in L$, one may present an arbitrary element of $U(L)$ as a linear combination of words

$$
\tilde{c}_{1} \dashv \tilde{c}_{2} \dashv \cdots \dashv \tilde{c}_{n}, \quad c_{i} \in B .
$$


Since $x \dashv \tilde{b}_{j}=0$ for any $x \in U(L)$, we may assume $c_{2}, \ldots, c_{n} \in\left\{a_{i} \mid i \in I\right\}$. It remains to note that if $i_{1}>i_{2}$ then

$$
\begin{array}{r}
x \dashv\left(\tilde{a}_{i_{1}} \dashv \tilde{a}_{i_{2}}\right)=x \dashv\left(\tilde{a}_{i_{2}} \dashv \tilde{a}_{i_{1}}\right)+x \dashv\left(\tilde{a}_{i_{1}} \dashv \tilde{a}_{i_{2}}-\tilde{a}_{i_{1}} \vdash \tilde{a}_{i_{2}}\right)+x \dashv \widetilde{\left[a_{i_{1}} a_{i_{2}}\right]} \\
=x \dashv\left(\tilde{a}_{i_{2}} \dashv \tilde{a}_{i_{1}}\right)+x \dashv\left[\widetilde{a_{i_{1}} a_{i_{2}}}\right] .
\end{array}
$$

Therefore, an arbitrary element of $U(L)$ can be presented as a linear combination of (11).

To complete the proof it is enough to show that the images of the elements (11) under $U\left(\rho_{U}\right)$ are linearly independent. Suppose $u$ is a word of the form (11). Compute $\left(U\left(\rho_{U}\right)(u)_{(z)} 1\right) \in M$ for $1 \in U\left(L^{\text {alg }}\right)$. Since $U\left(\rho_{U}\right)$ is a homomorphism of dialgebras,

$$
w=U\left(\rho_{U}\right)(u)=\left\{\tilde{c}_{(0)}\left\{\tilde{a}_{i_{1}(0)} \ldots\left\{\tilde{a}_{i_{n-1}(0)} \tilde{a}_{i_{n}}\right\} \ldots\right\}\right\} .
$$

Hence, relation (9) implies

$$
\left(w_{(z)} 1\right)=\left(\tilde{c}_{(z)}\left(\tilde{a}_{i_{1}(0)}\left(\cdots_{(0)}\left(\tilde{a}_{i_{n}(0)} 1\right) \ldots\right)\right)\right),
$$

which is easy to compute by (10):

$$
\left(w_{(z)} 1\right)=\tilde{c}_{(z)}\left(\bar{a}_{i_{1}} \ldots \bar{a}_{i_{n}}\right)=\bar{c} \bar{a}_{i_{1}} \ldots \bar{a}_{i_{n}}+z\left(c \otimes \bar{a}_{i_{1}} \ldots \bar{a}_{i_{n}}\right) .
$$

The coefficients at $z$ obtained are linearly independent in $M$ for different words of the form (11). Hence, the images of these words under $U\left(\rho_{U}\right)$ are also linearly independent.

Corollary 8 (PBW-Theorem for Leibniz algebras $[5,11])$. The universal enveloping associative dialgebra $U(L)$ for Leibniz algebra $L$ is isomorphic to $L \otimes U\left(L^{\text {alg }}\right)$ as a linear space.

\section{REFERENCES}

[1] Kolesnikov P. S., Varieties of dialgebras and conformal algebras, arXiv:math/0611501 3

[2] Kac V. G., Vertex Algebras for Beginners. University Lecture Series, vol. 10, AMS, Providence, RI, 1996.

[3] Belavin A. A., Polyakov A. M., Zamolodchikov A. B., Infinite conformal symmetry in twodimensional quantum field theory, Nuclear Phys. 241 (1984) 333-380.

[4] Loday J.-L., Pirashvili T., Universal enveloping algebras of Leibniz algebras and homology, Math. Ann. 296 (1993) 139-158.

[5] Loday J.-L., Dialgebras, in: Dialgebras and Related Operads. Lecture Notes in Mathematics, vol. 1763. Springer Verl., Berlin, 2001, pp. 7-66.

[6] Liu D., Steinberg - Leibniz algebras and superalgebras, J. Algebra 283 (1) (2005) 199-221.

[7] Sweedler M., Hopf Algebras, New York, Benjamin, 1969.

[8] Kac V. G., Formal distribution algebras and conformal algebras, Proc. XII-th International Congress in Mathematical Physics (ICMP'97), International Press, Cambridge, MA, 1999, pp. 80-97.

[9] Bakalov B., D'Andrea A., Kac V. G., Theory of finite pseudoalgebras, Adv. Math. 162 (1) (2001) 1-140.

[10] Kolesnikov P. S., Identities of conformal algebras and pseudoalgebras, Comm. Algebra 34 (6) (2006) 1965-1979.

[11] Aymon M., Grivel P.-P., Un théorème de Poincaré-Birkhoff-Witt pour les algèbres de Leibniz, Comm. Algebra 31 (2) (2003) 527-544.

Sobolev Institute of Mathematics, Novosibirsk, Russia

E-mail address: pavelsk@math.nsc.ru 